\title{
The Gini coefficient as a useful measure of malaria inequality among populations
}

\author{
Jonathan Abeles* and David J. Conway * (1)
}

\begin{abstract}
Background: Understanding inequality in infectious disease burden requires clear and unbiased indicators. The Gini coefficient, conventionally used as a macroeconomic descriptor of inequality, is potentially useful to quantify epidemiological heterogeneity. With a potential range from 0 (all populations equal) to 1 (populations having maximal differences), this coefficient is used here to show the extent and persistence of inequality of malaria infection burden at a wide variety of population levels.
\end{abstract}

Methods: First, the Gini coefficient was applied to quantify variation among World Health Organization world regions for malaria and other major global health problems. Malaria heterogeneity was then measured among countries within the geographical sub-region where burden is greatest, among the major administrative divisions in several of these countries, and among selected local communities. Data were analysed from previous research studies, national surveys, and global reports, and Gini coefficients were calculated together with confidence intervals using bootstrap resampling methods.

Results: Malaria showed a very high level of inequality among the world regions (Gini coefficient, $G=0.77$, 95\% $\mathrm{Cl}$ 0.66-0.81), more extreme than for any of the other major global health problems compared at this level. Within the most highly endemic geographical sub-region, there was substantial inequality in estimated malaria incidence among countries of West Africa, which did not decrease between 2010 ( $G=0.28,95 \% \mathrm{Cl} 0.19-0.36)$ and 2018 $(G=0.31,0.22-0.39)$. There was a high level of sub-national variation in prevalence among states within Nigeria $(\mathrm{G}=0.30,95 \% \mathrm{Cl} 0.26-0.35)$, contrasting with more moderate variation within Ghana ( $\mathrm{G}=0.18,95 \% \mathrm{Cl} 0.12-0.25)$ and Sierra Leone $(\mathrm{G}=0.17,95 \% \mathrm{Cl} 0.12-0.22)$. There was also significant inequality in prevalence among local village communities, generally more marked during dry seasons when there was lower mean prevalence. The Gini coefficient correlated strongly with the standard coefficient of variation, which has no finite range.

Conclusions: The Gini coefficient is a useful descriptor of epidemiological inequality at all population levels, with confidence intervals and interpretable bounds. Wider use of the coefficient would give broader understanding of malaria heterogeneity revealed by multiple types of studies, surveys and reports, providing more accessible insight from available data.

Keywords: Inequality, Epidemiology, Heterogeneity, Statistical index, Infection, Prevalence, Public helath

*Correspondence: abelesjonathan@gmail.com; david.conway@lshtm.ac.uk Faculty of Infectious and Tropical Diseases, London School of Hygiene and Tropical Medicine, Keppel Street, London WC1E 7HT, UK

\section{Background}

Describing levels of inequality of disease burden among populations is vital for epidemiology and global health, to highlight those who are affected disproportionately, and better target control interventions [1]. Most infectious disease and epidemiological reports do not give clear quantitative overviews on inequality, and the topic has 
been noted as requiring more attention in pursuit of the United Nations Sustainable Development Goals (UNSDGs) [2]. The particular UNSDG focusing on health includes an aim to end malaria as a public health problem by the year 2030 [3], encouraging control efforts, surveillance, and estimates of the situation through the World Health Organization (WHO) annual World Malaria Reports as well as national Malaria Indicator Surveys [4].

The Gini coefficient, an index widely used to describe income inequality, has been utilized previously to analyse general global health inequality $[5,6]$, sub-national differences in mortality [7], and for ecological studies [8], but only rarely for specific infectious diseases $[9,10]$. Disease burden variation among populations is more commonly presented using general measures of dispersion such as interquartile range, standard deviation, and sometimes the coefficient of variation (a scale-invariant coefficient obtained by dividing the standard deviation by the mean) [11]. However, these typical descriptors do not easily facilitate comparisons, whereas a benefit in using the Gini coefficient of inequality is that it has a standardized range from 0 (all populations equal) to 1 (populations having maximal differences) so that levels of inequality can be benchmarked.

Here the Gini coefficient is applied to illustrate inequality in malaria at several population levels, global, regional, sub-national and local. This shows that global inequity is higher than for other diseases, and is not decreasing in the areas that are most highly affected, while there are significant differences among countries in levels of subnational inequity, and local as well as seasonal variation.

\section{Methods}

\section{Gini coefficient of inequality}

The Gini coefficient is a measure of variation among different populations or groups, either of a positive resource or of an undesired burden, that is derived from the Lorenz curve of inequality [12]. In macroeconomics, it compares the proportion of 'wealth' owned by each single sub-population or group and describes the resulting inequality when considering the total. Applied to malaria or other diseases, it can be focused on comparing data at any level, for example among geographical regions globally or among local communities within a defined area. In such analysis, the Lorenz curve estimates how the distribution of disease burden (or other relevant measure such as infection prevalence) deviates from a theoretical line of perfect equality, and this deviation is summarized in the Gini coefficient. A coefficient of 1 is 'perfectly unequal', whereas a zero value represents 'perfectly equal' distribution.

The Gini coefficient (G) is estimated by comparing the values of the relevant indicator (such as prevalence) among all populations, and calculating all pairwise differences among them,

$$
G=\frac{\sum_{i=1}^{n} \sum_{j=1}^{n}\left|x_{i}-x_{j}\right|}{2 n^{2} \mu}
$$

using the following formula:

where $x_{i}$ is the value for each individual population $i$, and $x_{j}$ is the value for each of the individual populations $\mathrm{j}$ with which it is compared pairwise, there are n populations and $\mu$ is the mean value across all populations.

Gini coefficients were calculated in this study using STATA Version 15.3, with replicate analyses performed using Microsoft Excel and $\mathrm{R}$ version 3.6.3 to ensure consistency. Confidence intervals around the Gini coefficient were calculated using bootstrap resampling [13]. Equal bootstrap samples of size $\mathrm{n}$ are repeatedly drawn by sampling, and data are replaced after each sample. Bootstrap confidence intervals were calculated using $\mathrm{R}$ version 3.6.3, using command scripts as detailed in Additional file 1: Text S1, with resampling of $\mathrm{k}=500$ replicates as there is no significant benefit in using higher values of $\mathrm{k}$ in this context [14].

\section{Global and regional estimates for malaria and other public health challenges}

Variation in disease burden across the different WHO world regions (African Region, Region of the Americas, South-East Asia Region, European Region, Eastern Mediterranean Region, and Western Pacific Region) was first analysed using estimates of numbers of cases in the most recent WHO annual report or fact sheet for each global health problem. Malaria was analysed [4], as well as tuberculosis [15], HIV/AIDS [16], and Hepatitis C infection [17] representing major global infectious diseases, and the four groups of non-communicable diseases with highest overall mortality (cancer, respiratory disease, cardiovascular disease, and diabetes) $[18-20]$. Data were expressed as estimated number per 100,000 of the population in each region before calculating Gini coefficients (Additional file 1: Table S1). Malaria estimates were also presented as a percentage proportion of population at risk yearly between 2010 and 2018 (Additional file 1: Table S2).

The Gini coefficient was also calculated for variation across 16 West African countries using World Malaria Report estimates of numbers of cases for each year between 2010 and 2018 (Additional file 1: Table S3) [4], based on estimated numbers of cases in proportion to the number of people in areas of risk. 


\section{Malaria data at national, sub-national and local levels}

Recent national Malaria Indicator Surveys (MIS) in four West African countries (Nigeria, Ghana, Sierra Leone and Burkina Faso) were analysed to compare the levels of sub-national variation in heavily affected countries [2124]. Data were analysed from surveys of infection prevalence in under 5 year old children in 35 States in Nigeria (excluding one state that did not have sufficient data) [21], 10 Regions as previously defined in Ghana [22], 13 Regions in Burkina Faso [23], and 14 Districts in Sierra Leone [24]. Sub-national prevalence measurements from the MIS data analysed are tabulated in Additional file 1: Table S4.

To illustrate local variation in malaria infection prevalence and investigate temporal variation in highly endemic communities, data from The Garki Project were examined, given the availability of data from multiple cross-sectional surveys of multiple local village communities in an area of high prevalence in northern Nigeria in the early 1970s [25], from the archived database (https://garkiproject.nd.edu/). This analysis focused on an 18-month pre-intervention phase of the study during which eight successive parasitological cross-sectional surveys were conducted approximately every 10 weeks in each of 16 villages (data on a further six villages were not analysed as they were not surveyed at all eight rounds). Presence of $P$. falciparum was determined by microscopy and village-specific prevalence of $P$. falciparum at each survey is shown in Additional file 1: Table S5, after extraction from the archived project database. Data from a more recent study from The Gambia were also analysed for comparison, incorporating 20 villages with similar malaria seasonality [26], with variation in percent prevalence of $P$. falciparum determined by microscopy compared among villages in wet and dry seasons. Gini coefficient estimation from these and other previous data was performed using STATA version 15.3 with bootstrap confidence intervals calculated using $\mathrm{R}$ version 3.6.3.

\section{Results}

\section{Global variation in major health problems}

Across the six WHO world regions, inequality was greater for each of the infectious diseases compared to the major non-communicable diseases (Fig. 1). There was most extreme inequality in malaria burden (Gini coefficient, $\mathrm{G}=0.77,95 \%$ CI $0.66-0.81$ ), which was significantly higher than for each of the other diseases as demonstrated by bootstrap confidence intervals (Fig. 1 and Additional file 1: Table S1).

Removing the Europe WHO region from the malaria calculation (which had no reported cases in 2018) did not greatly reduce the index of inequality $(G=0.73,95 \%$

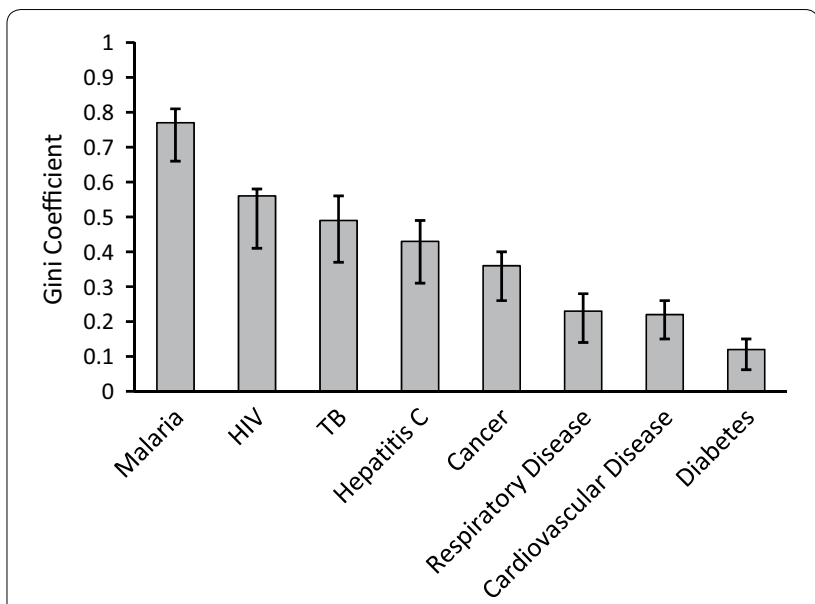

Fig. 1 Exceptionally high global inequality of malaria burden compared with other major public health indices. Gini coefficients of inequality of disease burdens among the six major regions of the world were calculated for eight major public health problems. The world regions are Africa, The Americas, South East Asia, Europe, Western Pacific, and Eastern Mediterranean as defined by the World Health Organization (WHO). Analyses are based on data or estimates extracted from the most recent fact sheets or world reports, or Global Health Estimates by WHO (listed in Additional file 1:Table S1). Malaria, HIV, and tuberculosis (TB) estimates represent new infections in 2018 $[4,15,16]$, while Hepatitis $C$ estimates represent new infections in 2015 [17]. For the four non-communicable diseases with highest mortality, different types of estimates are used as examples: diabetes estimates were based on prevalence in adults in 2014 [18], cancer estimates refer to new cases in the year of 2018 [19], while estimates for cardiovascular and respiratory disease refer to attributable deaths in 2016 [20]. The inequality of malaria burden was higher than for the other indices, as shown by the Gini coefficient estimates (with 95\% bootstrap confidence intervals)

CI 0.59-0.77). Removing the African WHO region (containing approximately $90 \%$ of all malaria cases), showed residual inequality among remaining regions to be much lower but still substantial ( $\mathrm{G}=0.40,95 \%$ CI $0.26-0.54)$, reflecting that most other global cases are in Asia or the Western Pacific. There was no decline in levels of global inequality between 2010 and 2018 based on data estimates from the World Malaria Report ( $G$ values for each year remained between 0.76 and 0.78 , Additional file 1 : Table S2).

\section{Variation in malaria within West Africa}

As the African region has the majority of the malaria burden, and more than half of the cases are estimated to occur in West Africa, we analysed the inequality among the 16 countries that constitute The West African subregion according to the UN definition. This shows the variation in estimated malaria burden as a proportion of overall populations among countries in West Africa between 2010 and 2018, and the persistent inequality is revealed by the Gini coefficient (Fig. 2 and Additional 


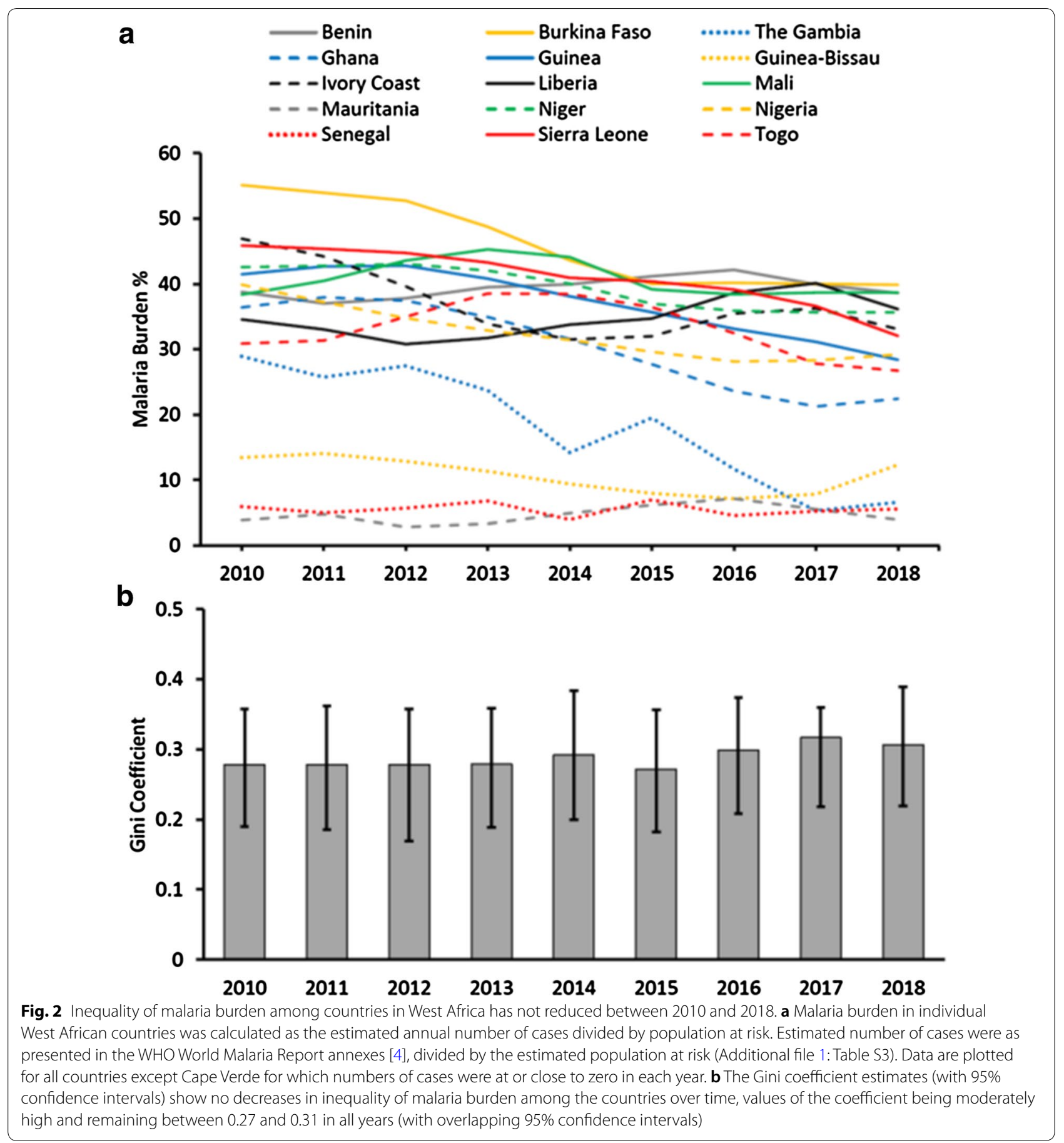

file 1: Table S3). Although there have been moderate reductions in malaria overall, and notable reductions in a few countries, the average burden is still high and variation among countries persists (Fig. 2a). Accordingly, the Gini Coefficient of inequality remained high, between 0.27 and 0.32 in each year (Fig. 2b). In 2018, the final year estimated, there was still marked inequality in malaria burden among countries in West Africa $(G=0.31,95 \%$
CI 0.22-0.39) and no indication of this having reduced from 2010 onwards.

\section{Sub-national variation in malaria within high burden countries in West Africa}

Sub-national variation within four of the highest burden countries was analysed using Malaria Indicator Survey data of malaria infection prevalence in children under 
5 years of age, with surveys between the years of 2014 and 2016 allowing prevalence to be analysed for each of the principal formal administrative divisions within each country (States in Nigeria, Regions in Ghana and Burkina Faso, Districts in Sierra Leone) (Fig. 3 and Additional file 1: Table S4). Sierra Leone had the highest mean prevalence, but the highest level of sub-national inequality in malaria parasite prevalence was seen in Nigeria $(G=0.30$, 95\% CI 0.26-0.35), followed by Burkina Faso $(G=0.25$, 95\% CI 0.19-0.29), with more moderate sub-national inequality within Ghana $(\mathrm{G}=0.18,95 \% \mathrm{CI} 0.12-0.25)$ and Sierra Leone $(G=0.17,95 \%$ CI $0.12-0.22)$.

\section{Seasonal malaria heterogeneity among villages in a highly endemic area}

Local variation was investigated among villages in the Garki Project, a large study conducted in northern Nigeria in the early $1970 \mathrm{~s}$ [25]. The $P$. falciparum prevalence was compared among 16 villages for which there were data at eight different survey timepoints (each survey separated by approximately 10 weeks) during the preintervention phase of the project (Fig. 4a and Additional file 1: Table S5). An overall seasonal peak of malaria prevalence is evident which corresponds to the late wet season and immediate post-wet season (surveys 5 and 6), while lower prevalence is seen during the annual dry seasons (surveys 2 and 3 for one year, and surveys 7 and 8 for the following year). The Gini coefficients are highest at survey timepoints 2,3 , and 7 , which coincide with the dry seasons (Fig. 4b). This demonstrates the ability of the Gini coefficient to track local variation in epidemiology, including temporal changes and effects of seasonality. To test for consistency in variation among villages across years, the rank order of prevalence in villages at survey 2 and survey 7 (representing similar points in consecutive dry seasons) was tested and shown to be significantly correlated (Spearman's rho $=0.55, \mathrm{P}=0.028$ ). This indicates that a significant component of the inter-village variation was maintained for at least a year.

Data were then analysed from a study of 20 villages in The Gambia [26], where malaria endemicity is lower and surveys were conducted shortly after malaria had declined significantly throughout the country [27, 28]. Comparing across all villages surveyed using the data provided in the original publication, heterogeneity was slightly higher in the dry season $(G=0.55)$ than the wet season $(G=0.40)$ (Additional file 1: Figure $S 1$ ). Focusing on the eastern part of The Gambia where malaria prevalence is highest also showed greater variation among villages in the dry season compared to the wet season (Additional file 1: Figure S1). Variation among villages was generally higher than seen in the data from The Garki Project.

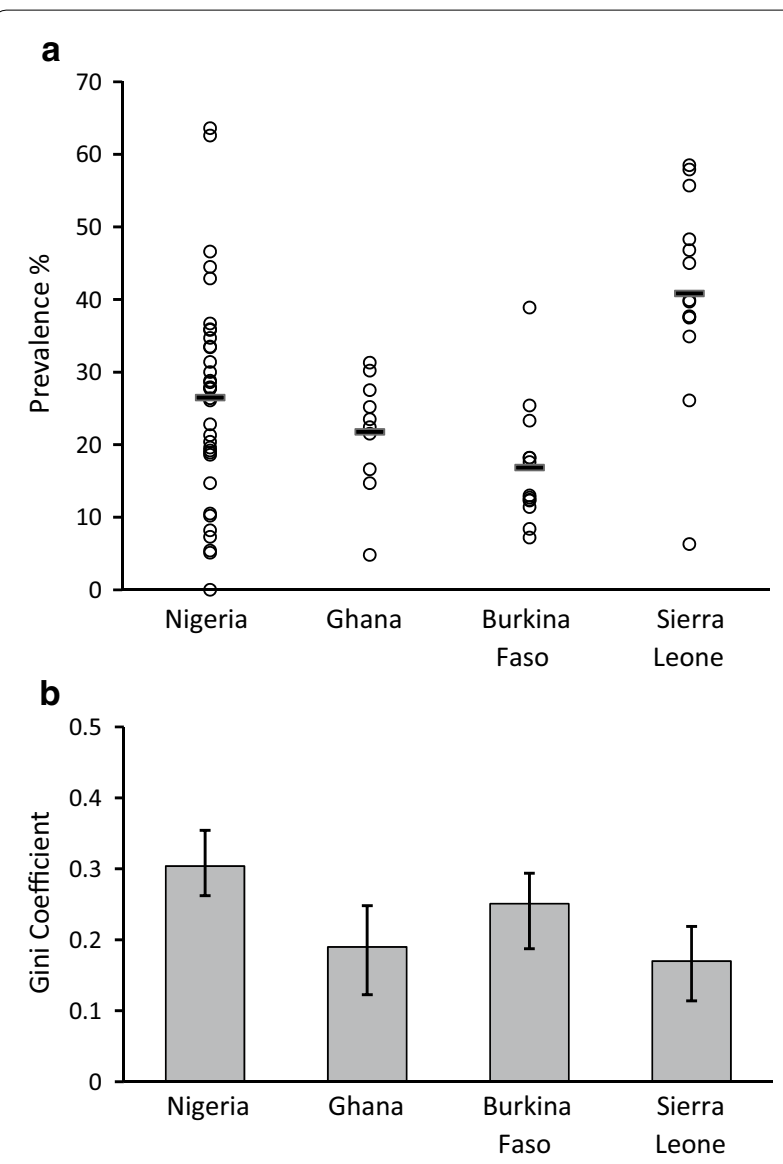

Fig. 3 Inequality of malaria prevalence among major administrative regions within four high burden countries in West Africa. a Each point represents the community prevalence of slide-positive malaria infection in children 6-59 months of age in the major administrative geographical regions within each of four countries as reported their most recent malaria indicator surveys (MIS). Data are analysed for 35 States in Nigeria from the 2015 MIS (having excluded one that did not have sufficient data) [21], 10 Regions within Ghana from the 2016 MIS [22], 13 Regions in Burkina Faso from the 2017 MIS [23], and 14 Districts in Sierra Leone from the 2016 MIS [24], as presented in Additional file 1: Table S4. b. The within-country Gini coefficient of inequality was highest for Nigeria $(G=0.30,95 \% \mathrm{Cl} 0.26-0.35)$, indicating a similar amount of variation within that country as exists among all 16 countries of West Africa. This was significantly greater variation than within Ghana or Sierra Leone

\section{Comparison to the coefficient of variation}

There is a strong correlation between the values of the Gini Coefficient presented here, and the values of the Coefficient of Variation (CV, standard deviation divided by the mean) calculated for each of the same datasets $\left(\right.$ Spearman's rho $\left.=0.982, \mathrm{P}<10^{-4}\right)$. Gini coefficient values ranged from 0.05 to 0.77 , while $C V$ values ranged from 9 to $198 \%$, with a strong correlation over the whole range (Fig. 5). As the Gini coefficient is bounded between 0 and 1 and has bootstrap confidence intervals (not plotted in 
Fig. 5), it offers advantages for interpretation compared to the use of an unbounded CV index.

\section{Discussion}

Inequality in malaria burden among populations is effectively summarized into a single index using the Gini coefficient, as shown here. Among leading global infectious and non-communicable public health problems, malaria shows the highest amount of inequality among different world regions, with a Gini coefficient of 0.77 being closer to the theoretically maximum possible value of 1.0 than to zero which would indicate equitable distribution. This coefficient has not been reduced in recent years, so there clearly needs to be increased effort to reducing the malaria burden in the most highly affected African region, while sustaining recent reductions of malaria elsewhere. This global need is already qualitatively clear [4], but the use of the Gini coefficient highlights the extreme situation for malaria in comparison to other diseases, and shows the measurability of inequality which is essential for future monitoring of progress.

Of equal importance, the Gini coefficient is also shown to be useful for summarising inequality at other population levels, from regional to local. Within West Africa, the sub-region with the highest overall malaria burden globally, the coefficient shows that malaria inequality among countries has not declined in recent years, reflecting that relative reductions in malaria burden have not been particularly great in the countries with most malaria. Moreover, the amount of sub-national inequality within four high burden countries in West Africa is also shown to be significantly variable. For example, there is more inequality in the infection prevalence among different states in Nigeria than among the major administrative areas within Ghana or Sierra Leone, analysing data from national Malaria Indicator Surveys that employ broadly comparable survey methods. The causes of such sub-national inequality will be complex and require more research attention for future malaria control.

The Gini coefficient is sensitive to village-level, arealevel, and seasonal variation, as illustrated here by reanalyses of research survey data from studies previously conducted in different parts of West Africa. The coefficient has features that make it potentially a more useful descriptor of epidemiological heterogeneity than other summary indices. The Gini coefficient demonstrates a defined lower and upper boundary of 0 (perfect equality) and 1 (perfect inequality) while the coefficient of variation $(\mathrm{CV})$ based on standard deviation does not. Although there is a strong correlation in their quantification of heterogeneity, the CV summarizes variation in an unbounded range that can transcend $100 \%$. The Gini coefficient is therefore more appropriate for use in the

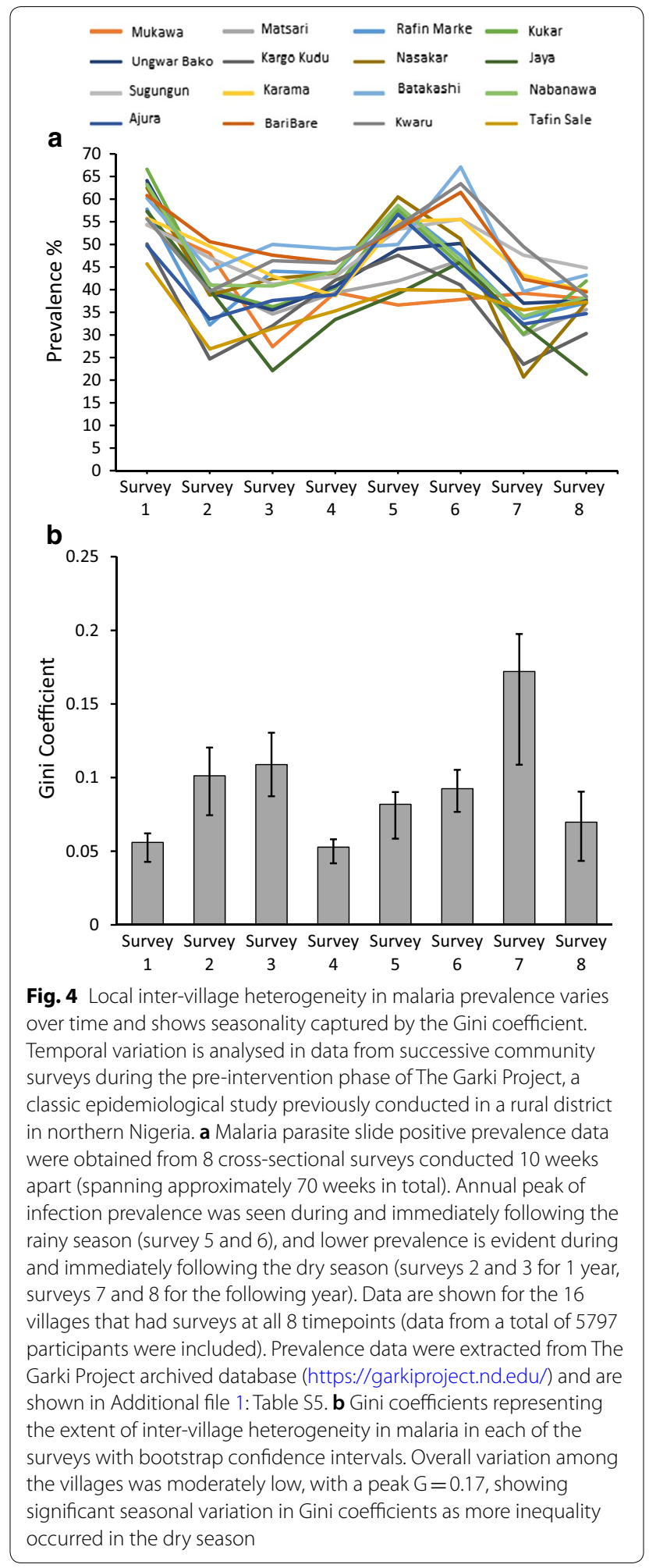

context of epidemiological studies and disease reports, to enable a more standardized quantitative interpretation of inequality. 


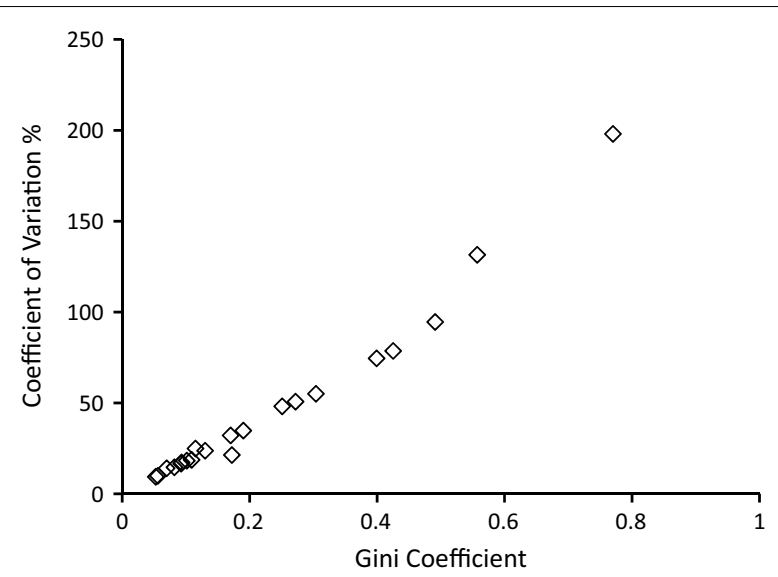

Fig. 5 Gini Coefficient compared to Coefficient of Variation for inter-population comparisons. This shows strong correlation between the Gini coefficient and the standard coefficient of variation (standard deviation divided by the mean) illustrating the Gini coefficient as a robust general statistical descriptor of variation. This is alongside its interpretive utility as being bounded between 0 and 1 (whereas the coefficient of variation is an unbounded proportional value that does not offer an intuitive scale for epidemiological comparisons). There were 21 values compared for each index here, representing data from different analyses presented in this paper: eight for various diseases among world health regions (from Fig. 1), one for malaria across the countries of West Africa (Fig. 2), four of sub-national variation in malaria prevalence in selected West African countries (Fig. 3), and eight sequential surveys across villages in The Garki Project (Fig. 4)

While the Gini coefficient is a useful descriptor, limitations should be considered. Technically, although bootstrap resampling is a generally robust method of calculating confidence intervals for the Gini coefficient, it has been suggested that in small samples of uniform, normal, or lognormal distributions bootstrap confidence intervals may be calculated as too narrow [14, 29], and robustness of these intervals increases with larger numbers of sampled populations. Statistical methods have been developed to mitigate this issue by approximating the Lorenz curve of a log-normal distribution [30], and could be investigated in future to check the sensitivity of confidence interval estimations. Also, although this was not a particular issue with the data analysed here, Gini coefficients could be skewed by 'small number bias' if they were based on samples of populations with extremely low prevalence, essentially corresponding to sampling noise that gives a systematic bias towards an inflated Gini coefficient in such situations.

Epidemiologically, the Gini coefficient needs to be recognized as a simple relative measure that does not present absolute differences, and different distributions of measurements may produce the same Gini coefficient. Demographic and socioeconomic differences, as well as ecological, genetic and geographical determinants all combine together $[31,32]$ and the effect of each is not explored or separately accounted for by the Gini coefficient. Indeed, in its more usual application to economic inequality, it has been well pointed out that use of the Gini coefficient should not preclude other analyses that can help understand details of the underlying heterogeneity [33]. Clearly, the coefficient does not substitute for separate analyses of hypothetical epidemiological determinants, or for maps of disease distribution, where these are available or where they may be estimated [34, 35]. Instead, it should be applied alongside presentation of more detailed or qualitative data, and used to advocate focus on populations most affected and where control of malaria is most needed, aiming to reduce the extreme inequity that continues to prevail at multiple levels.

\section{Conclusion}

The Gini coefficient is a useful index for descriptive epidemiology, particularly relevant for malaria, which shows exceptional levels of global inequality compared to other diseases. As illustrated here, it is applicable to a wide variety of data sources to show the degree of inequality among world regions, or countries within a region, as well as sub-nationally or locally. It should be more widely used in the presentation of prevalence data, disease burden reports, national indicator surveys, and for a broad range of population-based research.

\section{Supplementary information}

Supplementary information accompanies this paper at https://doi. org/10.1186/s12936-020-03489-x.

Additional file 1: Text S1. Calculating the Gini coefficient and bootstrap $95 \%$ confidence intervals using $R$ version 3.6.3. Table S1. Gini coefficients of inequality among $\mathrm{WHO}$ world regions in the estimated burden (per 100,000 of the population) of major global diseases. Table S2. No decline in the Gini coefficient of global malaria inequality based on estimated malaria cases as a percentage of Population at Risk between 2010 and 2018. Table S3. No decline in the Gini coefficient of malaria inequality among 16 West African countries based on estimated malaria cases as a percentage of Population at Risk between 2010 and 2018. Table S4. Gini coefficients show differences among West African countries in the levels of sub-national variation in malaria parasite prevalence in community surveys of children $<5$ years of age. Table S5. Gini coefficients show extent of variation in community prevalence of $P$. falciparum among 16 villages in the Garki Project in northern Nigeria in eight cross-sectional surveys during the pre-intervention phase of the project. Figure S1. High Gini coefficients of variation in P. falciparum prevalence among villages in The Gambia with seasonal malaria transmission. Variation was analysed among 20 villages each surveyed at two seasonal times.

\section{Acknowledgements}

We are grateful to colleagues who have discussed or commented on the analyses.

\section{Authors' contributions}

JA contributed to study design, performed analyses and co-wrote the manuscript. DJC conceived the study, contributed to study design, supervised 
analyses and co-wrote the manuscript. Both authors read and approved the final manuscript.

\section{Funding}

Research time of DJC is funded in part by the UK Medical Research Council (Grant MR/S009760/1).

\section{Availability of data and materials}

Previous epidemiological data analysed here are tabulated in Additional file or easily accessible from the cited sources, for which all references are given in the main text. The methods are fully given in the text and Additional file 1.

\section{Ethics approval and consent to participate}

The Research Governance and Integrity Office of the London School of Hygiene and Tropical Medicine approved this analysis (reference 17018), and declared it not to require separate review by the Ethics Committee as it involved secondary analyses of data already in the public domain.

\section{Consent for publication}

Not applicable.

\section{Competing interests}

The authors declare no competing interests.

Received: 7 September 2020 Accepted: 9 November 2020

Published online: 02 December 2020

\section{References}

1. Hosseinpoor AR, Bergen N, Schlotheuber A. Promoting health equity: WHO health inequality monitoring at global and national levels. Glob Health Action. 2015;8:29034.

2. Hosseinpoor AR, Bergen N, Schlotheuber A, Boerma T. National health inequality monitoring: current challenges and opportunities. Glob Health Action. 2018;11:1392216.

3. UN. Sustainable Development Goals. https://www.un.org/sustainabl edevelopment/sustainable-development-goals/; 2015.

4. WHO. World Malaria Report 2019. Geneva: World Health Organization 2019. https://www.who.int/publications/i/item/world-malaria-repor $\mathrm{t}-2019$.

5. Steinbeis F, Gotham D, von Philipsborn P, Stratil JM. Quantifying changes in global health inequality: the Gini and slope inequality indices applied to the global burden of disease data, 1990-2017. BMJ Glob Health. 2019;4:e001500

6. Asada Y, Hurley J, Norheim OF, Johri M. A three-stage approach to measuring health inequalities and inequities. Int J Equity Health. 2014;13:98.

7. Skaftun EK, Verguet S, Norheim OF, Johansson KA. Geographic health inequalities in Norway: a Gini analysis of cross-county differences in mortality from 1980 to 2014. Int J Equity Health. 2018;17:64.

8. Damgaard C, Weiner J. Describing inequality in plant size or fecundity. Ecology. 2000;81:1139-42.

9. Duarte EC, Ramalho WM, Tauil PL, Fontes CJ, Pang L. The changing distribution of malaria in the Brazilian Amazon, 2003-2004 and 2008-2009. Rev Soc Bras Med Trop. 2014;47:763-9.

10. Kerani RP, Handcock MS, Handsfield HH, Holmes KK. Comparative geographic concentrations of 4 sexually transmitted infections. Am J Public Health. 2005;95:324-30.

11. de Vries EF, Heijink R, Struijs JN, Baan CA. Unraveling the drivers of regional variation in healthcare spending by analyzing prevalent chronic diseases. BMC Health Serv Res. 2018;18:323.

12. Moskowitz CS, Seshan VE, Riedel ER, Begg CB. Estimating the empirical Lorenz curve and Gini coefficient in the presence of error with nested data. Stat Med. 2008:27:3191-208.
13. Efron B. The Jackknife, the Bootstrap and other resampling plans. Soc Indust Appl Mathe. 1982. https://doi.org/10.1137/1.9781611970319.

14. Dixon PM, Weiner J, Mitchell-Olds T, Woodley R. Bootstrapping the Gini coefficient of inequality. Ecology. 1987;68:1548-51.

15. WHO. Global Tuberculosis Report 2019. Geneva: World Health Organization; 2019.

16. WHO. HIV/AIDS Data and Statistics 2018. Geneva: World Health Organization; 2019.

17. WHO. Global Hepatitis Report 2017. Geneva: World Health Organization; 2017.

18. WHO. Global Report on Diabetes. W Geneva: World Health Organization; 2016.

19. Global Cancer Observatory. Cancer Incidence in Five Continents XI International Agency for Research on Cancer. Lyon: World Health Organization; 2017.

20. WHO. Global Health Estimates 2016. Geneva: World Health Organization; 2017.

21. National Malaria Elimination Programme and ICF. Nigeria Malaria Indicator Survey 2015. Abuja: Nigeria, and Rockville, USA; 2016.

22. Ghana Statistical Service (GSS), and ICF. Ghana Malaria Indicator Survey 2016. Accra, Ghana. 2017.

23. Institut National de la Statistique et de la Démographie (INSD), Programme National de Lutte contre le Paludisme (PNLP) and ICF. Enquête sur les indicateurs du paludisme au Burkina Faso, 2017-2018. Ouagadougou, Burkina Faso and Rockville, USA; 2018.

24. National Malaria Control Programme [Sierra Leone] and ICF. Sierra Leone Malaria Indicator Survey 2016. Freetown, Sierra Leone, Rockville, USA; 2016.

25. Molineaux L, Gramiccia G. The Garki Project. Geneva: World Health Organization; 1980.

26. Oduro AR, Conway DJ, Schellenberg D, Satoguina J, Greenwood BM, Bojang KA. Seroepidemiological and parasitological evaluation of the heterogeneity of malaria infection in the Gambia. Malar J. 2013;12:222.

27. Ceesay SJ, Casals-Pascual C, Erskine J, Anya SE, Duah NO, Fulford AJ, et al. Changes in malaria indices between 1999 and 2007 in The Gambia: a retrospective analysis. Lancet. 2008;372:1545-54.

28. Ceesay SJ, Casals-Pascual C, Nwakanma DC, Walther M, Gomez-Escobar $\mathrm{N}$, Fulford AJ, et al. Continued decline of malaria in The Gambia with implications for elimination. PLOS ONE. 2010;5:e12242.

29. Qin YS, Rao JNK, Wu CB. Empirical likelihood confidence intervals for the Gini measure of income inequality. Econ Model. 2010;27:1429-35.

30. Ultsch A, Lotsch J. A data science based standardized Gini index as a Lorenz dominance preserving measure of the inequality of distributions. PLoS ONE. 2017;12:e0181572.

31. Bousema T, Drakeley C. Determinants of malaria transmission at the population level. Cold Spring Harb Perspect Med. 2017;7:a025510.

32. White NJ, Pukrittayakamee S, Hien TT, Faiz MA, Mokuolu OA, Dondorp AM. Malaria Lancet. 2014;383:723-35.

33. Piketty T. Capitalism in the 21 st Century. Cambridge: Harvard University Press; 2014

34. Battle KE, Lucas TCD, Nguyen M, Howes RE, Nandi AK, Twohig KA et al. Mapping the global endemicity and clinical burden of Plasmodium vivax, 2000-17: a spatial and temporal modelling study. Lancet. 2019;394:332-43.

35. Weiss DJ, Lucas TCD, Nguyen M, Nandi AK, Bisanzio D, Battle KE, et al. Mapping the global prevalence, incidence, and mortality of Plasmodium falciparum, 2000-17: a spatial and temporal modelling study. Lancet. 2019;394:322-31.

\section{Publisher's Note}

Springer Nature remains neutral with regard to jurisdictional claims in published maps and institutional affiliations. 